\title{
Spin Evolution of Pulsars with Weakly Coupled Superfluid Interiors
}

\author{
Armen Sedrakian and James M. Cordes \\ Center for Radiophysics and Space Research, Cornell University, Ithaca, NY 14853
}

\begin{abstract}
We discuss the spin evolution of pulsars in the case where a superfluid component of the star is coupled to the observable crust on long, spindown timescales. The momentum transfer from the superfluid interior results in an apparent decay of the external torque and, after a dramatic increase, to an asymptotic decrease of the generic value of the braking index, e.g. $n=3$, to values $n \sim 2.5$ if the magnetic field of the star does not decay over its lifetime. In the case where an exponential decay of the magnetic field towards a residual value occurs, the star undergoes a spin-up phase after which it could emerge in the millisecond sector of the $P-\dot{P}$ diagram.
\end{abstract}

Subject headings: dense matter - magnetic fields - hydrodynamics - stars: interiors - stars: rotation - stars: neutron

The spin evolution of radio pulsars is governed by the braking torques acting on the solid component of the neutron star. A number of processes contribute to the energy losses, e.g. magnetic-dipole radiation (MDR) and a relativistic magnetohydrodynamic wind, which predict a braking torque $\propto-\gamma \Omega^{n}$, where $n$ is the braking index, $\gamma$ - a positive constant, whose explicit form depends on the emission process assumed. Timing observations provide the values of $n$ for four young pulsars (Kaspi et al. 1994; Boyd et al. 1995; Lyne, Pritchard, \& Graham-Smith 1988; Lyne et al. 1996), which fall in the range $1.4 \leq n \leq 2.84$, i.e. less than the canonical MDR value, $n=3$. It is generally agreed that the observed grouping of pulsars in the $P-\dot{P}$ plane requires torque evolution in terms of variation of a pulsar's magnetic moment. The precise form of the time-dependence is, however, unsettled. The possibilities considered range from magnetic field decay on timescales of order $10^{6}$ yr (Lyne, Manchester, \& Taylor 1985), decay on the same timescale towards a constant residual value several orders of magnitude lower than the initial one (Kulkarni 1986), to a marginal field decay (Romani 1990a). The separate class of millisecond pulsars, which have anomalously small braking torques, suggests that substantial torque decay occurs over 
the evolutionary timescale. Their locations in the small $P$ and small $\dot{P}$ part of the $P-\dot{P}$ diagram are commonly attributed to evolution in binary stellar systems, whether or not such pulsars are found in binaries today. A critical part of the evolution is the transfer of angular momentum and the attenuation of the magnetic field by accretion (Romani 1990b). Isolated millisecond pulsars may be accounted for by the evaporation of the companion by the pulsar's relativistic wind (Phinney et al. 1988; Ruderman, Shaham, \& Tavani 1991) or three-body break-up processes (Romani, Kulkarni, \& Blandford 1987). Detailed models suggest that evaporation may not operate quickly enough to account for all such objects. Though there is compelling evidence for the binary evolution scenario of millisecond pulsars, the existence of solitary objects among this class of pulsars appears somewhat enigmatic.

The present paper studies, phenomenologically, the secular evolution of a solitary pulsar with a small fraction of interior superfluid coupled to the normal component on evolutionary timescales $\tau_{s} \simeq P / 2 \dot{P} \sim 10^{3}-10^{6}$ yr. This assumption will be taken as a working hypothesis throughout most of the paper; the microscopic physics that might allow such a circumstance is discussed at the end of the paper. The implications of our hypothesis of weakly coupled superfluid interiors on the spin evolution in an accreting system are not discussed here.

We shall adopt a non-linear version of the familiar two-component model of superfluid rotator (Baym et al. 1969). The equations are first derived for an arbitrary configuration in equilibrium and are specified for the spherical case thereafter. The Euler equations for the superfluid and normal components in the two-fluid superfluid hydrodynamic (e.g. Khalatnikov 1965) read

$$
\begin{aligned}
\rho_{s}\left[\frac{\partial}{\partial t} \mathbf{v}_{s}+\left(\mathbf{v}_{s} \cdot \nabla\right) \cdot \mathbf{v}_{s}\right] & =-\frac{\rho_{s}}{\rho} \boldsymbol{\nabla} p-\rho_{s} \boldsymbol{\nabla} \varphi+\mathbf{f}^{(m f)} \\
\rho_{n}\left[\frac{\partial}{\partial t} \mathbf{v}_{n}+\left(\mathbf{v}_{n} \cdot \boldsymbol{\nabla}\right) \cdot \mathbf{v}_{n}\right] & =-\frac{\rho_{n}}{\rho} \boldsymbol{\nabla} p-\rho_{n} \boldsymbol{\nabla} \varphi-\mathbf{f}^{(m f)}+\mathbf{f}^{(e x)},
\end{aligned}
$$

where $\mathbf{v}$ and $\rho$ denote velocity and density; the subscripts $s$ and $n$ refer to the superfluid and the normal components; $p$ is the pressure; $\varphi$, the gravitational potential, and $\mathbf{f}^{(e x)}$, the external force acting on the normal component. It is assumed that the densities of the superfluid and normal components are conserved separately. Here the mutual friction force is written in general form as

$$
\mathbf{f}^{(m f)}=-\beta\left[\boldsymbol{\nu} \times\left[\boldsymbol{\omega} \times\left(\mathbf{v}_{n}-\mathbf{v}_{s}\right)\right]\right]-\beta^{\prime}\left[\boldsymbol{\omega} \times\left(\mathbf{v}_{n}-\mathbf{v}_{s}\right)\right],
$$

where $\boldsymbol{\omega}=\boldsymbol{\nabla} \times \mathbf{v}_{s}$ is the superfluid circulation; $\boldsymbol{\nu}=\boldsymbol{\omega} / \omega$; and the vortex tension forces $(\propto \boldsymbol{\nabla} \times \boldsymbol{\nu})$ are dropped (we also ignore the effects related to the lattice stress energy; see e.g. Chandler \& Baym 1986 for a discussion of these effects); the parameters $\beta, \beta^{\prime}$ 
are phenomenological mutual friction coefficients. The macroscopic equations of motion, derived from (1) and (2), assuming hydrostatic equilibrium, are

$$
\begin{aligned}
\frac{d}{d t}\left(I^{(s)} \delta_{i j}-I_{i j}^{(s)}\right) \Omega_{s j} & =K_{i}+K_{i}^{\prime} \\
\frac{d}{d t}\left(I^{(n)} \delta_{i j}-I_{i j}^{(n)}\right) \Omega_{n j} & =-K_{i}-K_{i}^{\prime}+K_{i}^{(e x)},
\end{aligned}
$$

where $\Omega_{s}$ and $\Omega_{n}$ are the angular velocities of the superfluid and the normal components and $I_{i j}^{(s, n)}=\int \rho_{(s / n)} x_{i} x_{j} d V$, are the moment of inertia tensors of these components; $\left(I^{(s / n)} \equiv \operatorname{Tr} I_{i j}^{(s / n)}\right)$. The internal torques $K, K^{\prime}$, are defined as (summation over repeated indices is assumed)

$$
\begin{aligned}
K_{i} & =-2 \beta\left|\Omega_{s}\right|\left[\nu_{i} \nu_{j}\left(I_{k j}^{(s)} \delta \Omega_{k}-I^{(s)} \delta \Omega_{j}\right)+\nu_{j} \nu_{k}\left(I_{i j}^{(s)} \delta \Omega_{k}-I_{k j}^{(s)} \delta \Omega_{i}\right)\right], \\
K_{i}^{\prime} & =2 \beta^{\prime}\left|\Omega_{s}\right| \nu_{k} \varepsilon_{i j l} I_{k l}^{(s)} \delta \Omega_{j},
\end{aligned}
$$

where $\delta \Omega \equiv \Omega_{n}-\Omega_{s}$ is the difference between the normal and superfluid angular velocity vectors. We suppose, further, that the neutron star decelerates under the action of magnetic dipole radiation, in which case $n=3$ and $\gamma=B_{\perp}^{2} R^{6} / 6 c^{3}$, where, $B_{\perp}$ is the surface magnetic induction component perpendicular to the pulsar's spin axis and $R$ is the radius of the corotating star-magnetosphere system. Finite size corrections to the dipole radiation reduce the braking index to values between two and three (Melatos 1997), but as a fiducial value, we adopt the MDR value, $n=3$. From now on we shall assume a spherical symmetry, in which case torque $K^{\prime}$ vanishes. The resulting coupled equations can be integrated numerically for known initial conditions. Note that the linear model of Baym et al (1969) is recovered form these equations by defining a time-independent relaxation time $\left(2\left|\Omega_{s}\right| \beta\right)^{-1}$.

Shortly after birth, a cooling neutron star is expected to develop superfluid regions which carry most of the angular momentum of the interior in the form of an array of vortices, each carrying a single quantum of vorticity. When vortices nucleate, the superfluid and normal components are corotating, which is the initial condition for the subsequent integration of equations of motion for secular dynamics. As the external torque acts on the outer crust of the neutron star, the resultant spin down is communicated to different shells in the superfluid on different timescales determined by values of $\beta$. Example evolutionary tracks of pulsars on the $P-\dot{P}$ diagram are shown in Fig. 1.

Initially, the normal component spins down under the sole action of the external torque; the superfluid component remains uncoupled and, therefore, preserves its angular momentum acquired from the corotation phase at vortex nucleation. The imbalance between the superfluid and the normal component eventually starts to relax after the star evolves to times comparable to the dynamical coupling time $\sim\left(\Omega_{0} \beta\right)^{-1}$, where $\Omega_{0}$ is the 


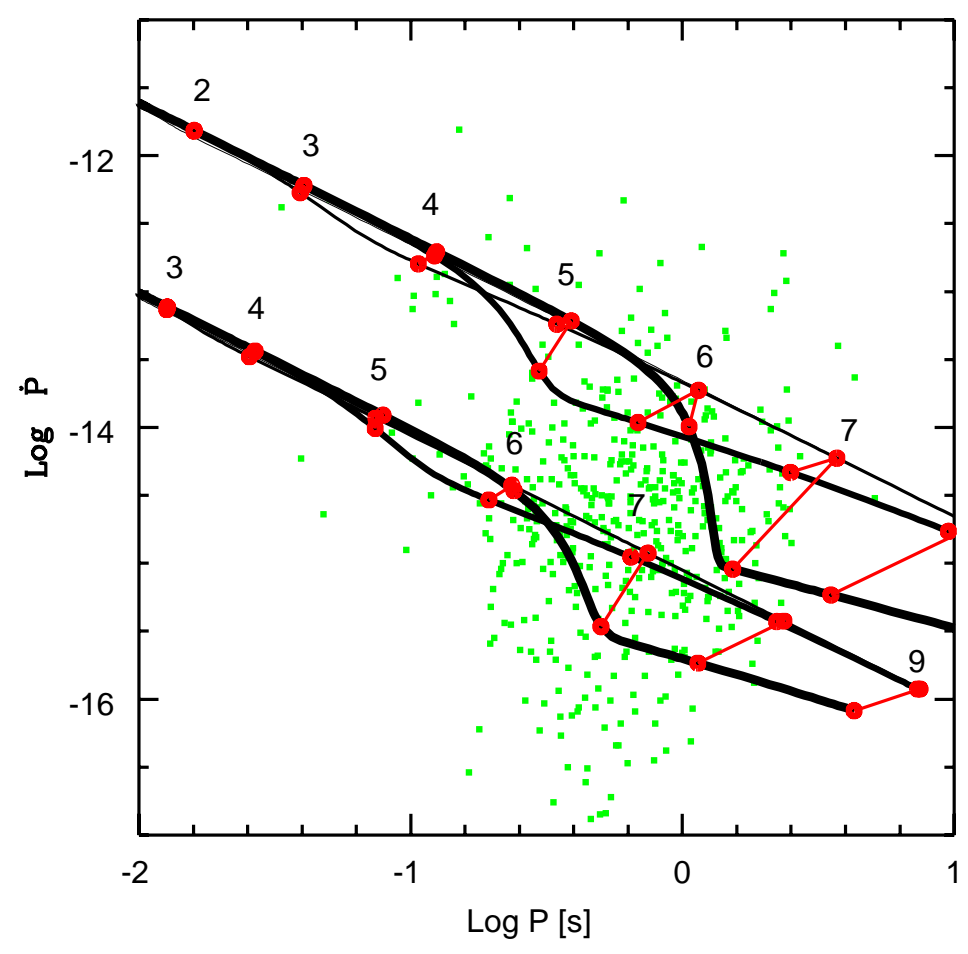

Fig. 1.- Evolutionary tracks of pulsars on the $P-\dot{P}$ diagram for magnetic field values of $B_{\perp}=10^{13}$ and $B_{\perp}=2 \times 10^{12}, \alpha=2 I^{(s)} / 3 I^{(n)}=0.1$ and $\log \beta=-14$ (thinnest line), $-16,-18$ (thickest line). The initial spin period is $10 \mathrm{msec}$. The labeling of the thick dots corresponds to $\log t$ (yr) with equal-time dots connected. 
initial corotation frequency. This is marked by the "drop" of the evolutionary tracks in the $P-\dot{P}$ diagram: the decrease of the angular momentum of the crust reduces due to momentum imparted from the interior. For values $\log \beta \geq-12$ (small coupling timescales) the star effectively maintains corotation between all superfluid and normal components. The slope of the tracks is then determined by the external torque and is, therefore, exactly the same as it would be for the rigid body system. However, if there is weakly coupled superfluid with large coupling timescales, there is a memory effect in that the star reaches a given point in the diagram later than in the rigid body case. For very small $\beta$ 's, the corotation phase is never achieved during the active lifetime of the pulsar, and the star crosses the deathline with $P, \dot{P}$ parameters radically different from the rigid-body casq $\boxplus$.

The time evolution of the apparent braking index is shown in Fig. 2. The deviations from the canonical rigid body, MDR value $(n=3)$ are nonmonotonic: the momentum impart from the superfluid causes a substantial increase of braking index because of the decrease in $\dot{P}(t)$ as a result of near cancellation of the external and internal torques on the dynamical coupling timescale. Subsequent evolution under the action of both internal and external torques implies braking indices in the range $\sim 2.5-2.8$, i.e. less than the canonical value 3 and in agreement with those deduced from the timing data; (these values are still considerably larger than the value $n=1.4$ reported for the Vela pulsar (Lyne et al 1996); the case of the Vela pulsar is, presumably, exceptional because of the high glitch activity of this pulsar). The evolution of $n(t)$ approaches the $n=3$ value asymptotically.

Relaxing the assumption of constant magnetic field, we next assume an exponential decay of the initial field to some residual value, i.e. we replace $B_{\perp} \rightarrow B_{\perp} \exp \left(-t / \tau_{d}\right)+B_{\perp}^{\text {res }}$, where $\tau_{d}$ is the decay constant, $B_{\perp}^{\text {res }}\left(\ll B_{\perp}\right)$ is the residual magnetic field value. The constant $B_{\perp}$ case is recovered in the limit $\tau_{d} \rightarrow \infty$; in this respect a smooth crossover between two version of magnetic field evolution is expected as $\tau_{d}$ is varied. In particular, one would expect that the power low field decay case, implying a slower field decay rate, would lead to a result intermediate between those considered here with no new qualitative modifications.

Simulations of the evolutionary tracks for $\tau_{d}=10^{6} \mathrm{yr}$ and $B_{\perp}=10^{13}$ are shown in Fig. 3. For fixed $\tau_{d}$ and $\log \beta \geq-12$ the evolution follows the rigid body case with an exponential decay on the timescale $\tau_{d}$. For $\log \beta=-16$ and -18 the slow coupling to the superfluid causes an initial drop of the tracks, which is followed by a zero crossing and a

\footnotetext{
${ }^{1}$ The theoretical deathline, beyond which the star turns off as radio pulsar, depends on the structure of surface magnetic and the radio-emission mechanism assumed, see K. Chen and M. Ruderman (1993); its precise location is, however, not needed for the present discussion.
} 


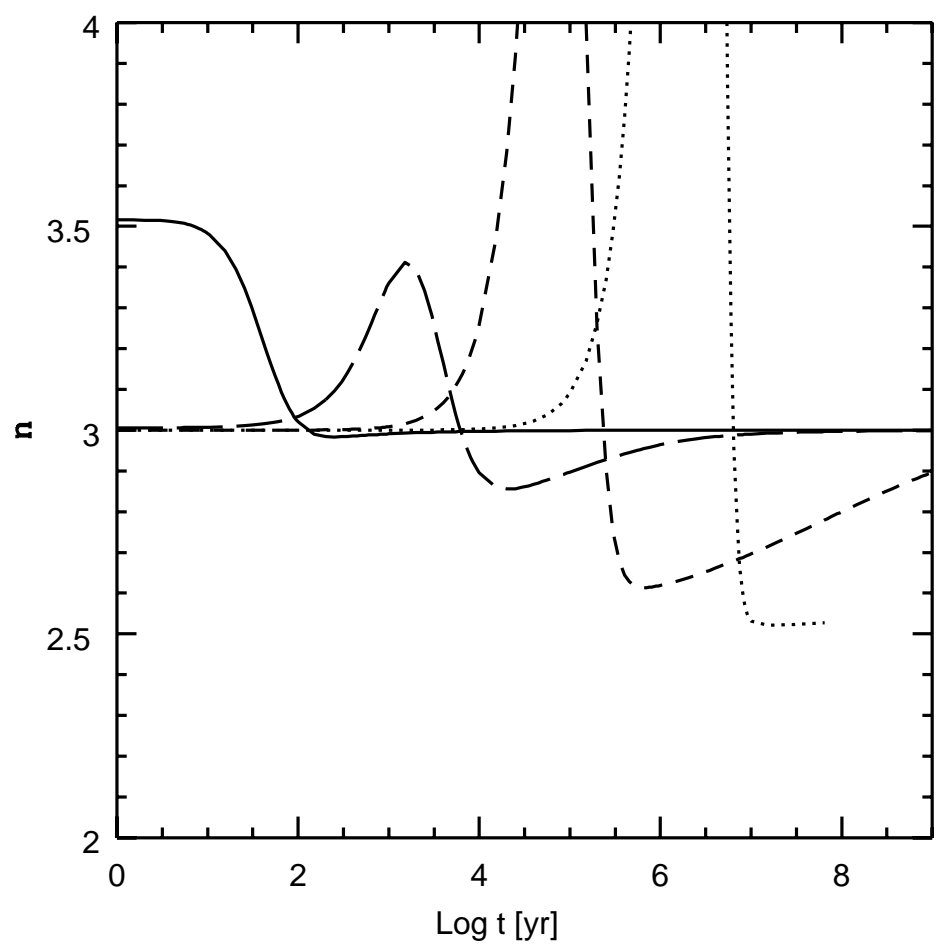

Fig. 2. - The time evolution of the braking index for $B_{\perp}=10^{13}, \alpha=0.1$; Solid, longdashed, dashed and dotted curves correspond to the values $\log \beta=-12,-14,-16,-18$. The initial spin period is $10 \mathrm{msec}$. 


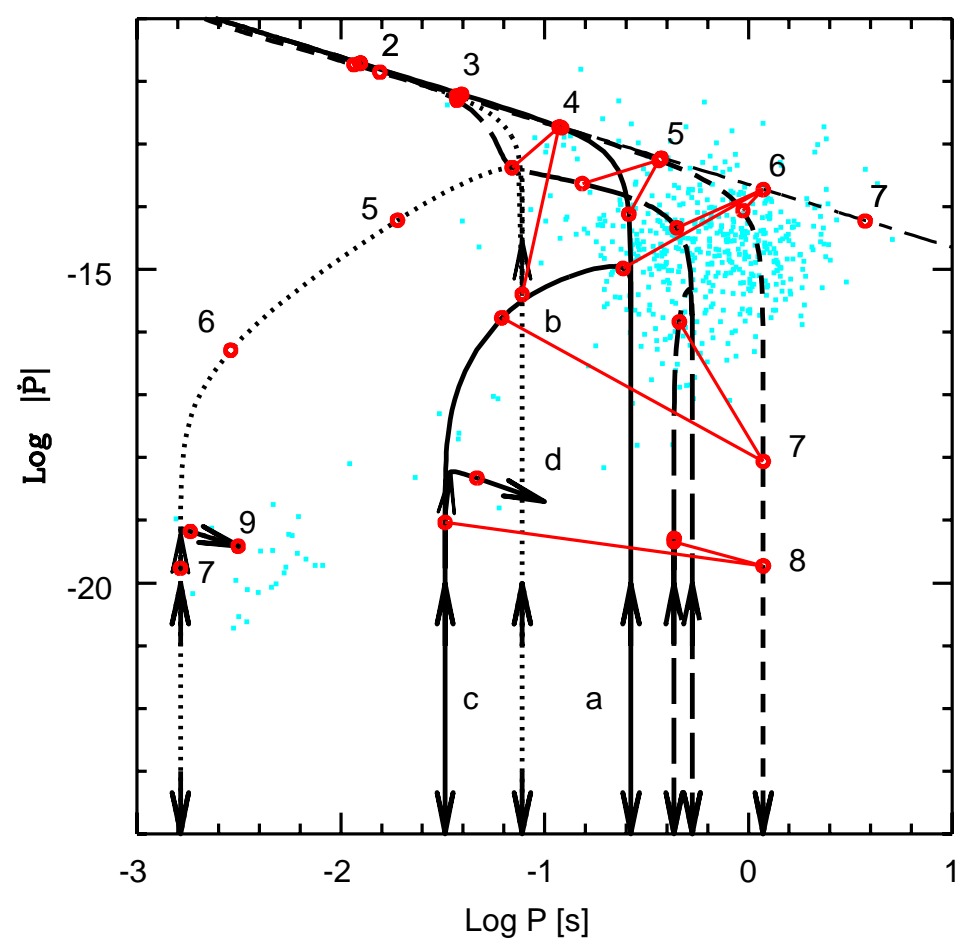

Fig. 3.- Evolutionary tracks of pulsars on the $P-|\dot{P}|$ diagram with magnetic field decay for initial magnetic field $B_{\perp}=10^{13}$ and residual field $B_{\perp}^{\text {res }}=10^{10} \mathrm{G}$. An initial period $1 \mathrm{~ms}$ is assumed, while $\alpha$ is fixed at the value 0.1. The dashed and dashed-dotted curves correspond to rigid body evolution with and without magnetic field decay. The long dashed and solid curves correspond to tracks with $\log \beta=-16$ and -18 respectively. The arrows show the direction of motion (see text). The dotted curve is the fit to PSR 1937+21 with parameters given in the text. Equal-time thick dots labeled by $\log t(\mathrm{yr})$ are connected in some cases; whenever the direction of motion is ambiguous these are supplied by arrows. 
spin-up; (the section labeled $a$ on the example track with $\log \beta=-18$ in Fig. 3). During the spin-up epoch (sections $a$ upwards $-b-c$ downwards) $\dot{P}<0$ since the angular momentum transferred to the crust is greater than that lost via dipole radiation. The spin-up epoch is restricted by the residual field, which causes the tracks to cross zero once more (section $c$ upwards), after which evolution enters a steady deceleration state (section $d$ ). When $\log \beta \leq-16$ the initial drop immediately goes over to a spin-up stage, because the external torque has decayed substantially before the coupling between the superfluid and the normal component sets in, [i.e. $\left.(\Omega \beta)^{-1} \geq \tau_{d}\right)$ ]. Note that the transitions from spin-down to spin-up and back to spin-down might require crossing the deathline: the pulsar will undergo period(s) of radio-silence, before eventually emerging as a millisecond pulsar. The possible set of parameters which fits the fastest known millisecond pulsar $1937+21$ is not uniquely defined; the generic requirement, which does not seem to conform the present view on the interior of neutron stars, is the large moment of inertia of the weakly coupled superfluid ( $\sim 60 \%$ of the total moment of inertia) and a fast field decay. In Fig. 3 the fit is obtained by setting the initial period to $0.8 \mathrm{~ms}, \alpha=1, \log \beta=-18, B_{\perp}^{\text {res }}=10^{9}$ and $\tau_{d}=10^{4}$ yr.

To what extent can values of the phenomenological parameters $\alpha$ and $\beta$ that produce spin up behavior be consistent with the microphysics of superfluid interiors? The $\alpha$ 's are fixed by prescribing the equation of state and the composition of the matter along with the density-profiles of pairing gaps. The $\beta$ 's, in the present model, are related to the local (microscopic) vortex-quasiparticle viscous friction coefficient $\eta$, via the relation $\beta=\eta \rho_{s} \omega /\left[\eta^{2}+\left(\rho_{s} \omega\right)^{2}\right]$. Of particular interest is the limit $\eta / \rho_{s} \omega \rightarrow \infty$, which shows that the strong coupling of quasiparticles to the vortex lattice on the local scale results in small values of $\beta$, long coupling timescales, and effectively weak coupling of the superfluid to the normal component on macro-scales. The term "weakly coupled superfluid" is used throughout the paper in the latter sense. The opposite limit, $\rho_{s} \omega / \eta \rightarrow \infty$, which also leads to weak coupling, seems to be less plausible.

Large friction and long coupling timescales in the superfluid crusts are predicted by the models of vortex creep (Link, Epstein \& Baym 1993, Alpar et al 1996), where the coupling depends exponentially on the temperature. In this case the secular and thermal evolutions are coupled (e.g. Shibazaki and Mochizuki 1995), $\beta$ is generally a function of time, and, therefore, our arguments apply only qualitatively. The minimal values of $\beta$ 's, inferred from the respective relaxation times, lie in the range $-12 \geq \log \beta_{\min } \geq-14$ for the time $\operatorname{span} 10^{4} \leq t \leq 10^{6} \mathrm{yr}$. For these values of $\beta$ and typical values $\alpha \leq 0.03$, the crust has a minor effect on the evolutionary tracks; a sizable effect, however, is expected for altering the apparent braking index (the value of $\alpha$ used in Fig. 2 is roughly by a factor 3.3-10 larger than that expected for the crusts, but this can be compensated by choosing a slightly smaller initial spin period). A possibility, that would introduce a new timescale 
in the problem, but is not incorporated in the present model, is the large scale motion of crustal matter and its superfluid phases (Ruderman 1991).

The dynamics of rotating superfluids in the quantum liquid core of a neutron star is well described in terms of the two-fluid hydrodynamics and eqs. (4), (5) are strictly applicable. Among several frictional processes (Sauls, Stein \& Serene 1982; Alpar, Langer, \& Sauls 1984, Sedrakian et al 1995) the largest values are found for the process of electron scattering off proton vortex clusters forming a substructure of the neutron vortex lattice (Sedrakian et al 1995). The minimal value of $\beta$ for this process is $\beta_{\min } \sim 10^{-18}$ at density $\rho_{\text {min }} \simeq 8.3 \times 10^{14} \mathrm{~g} \mathrm{~cm}^{-3}$, and corresponds to coupling times $\sim\left(2 \Omega_{0} \beta\right)^{-1}=2.5 \times 10^{6}$ yr for $\Omega_{0}=6283 \mathrm{~s}^{-1}$. This value places a lower theoretical constraint on the $\beta$-parameter for the processes we have examined $[$ ?

The BCS pairing would be suppressed when the velocity difference between the superfluid and the normal component exceeds the critical velocity $v_{\mathrm{cr}} \sim\left(\Delta / \epsilon_{F}\right) v_{F}$, where $\Delta$ is the gap, $\epsilon_{F}$ and $v_{F}$ are the Fermi energy and velocity. According to the familiar Landau criterion the quasiparticle excitations are spontaneously generated when the kinetic energy associated with the Galilean transformation to the moving frame exceeds the energy barrier $\sim 2 \Delta$. Similar criterion for Cooper pair disintegration is obtained when considering the four point nucleon-nucleon scattering vertex for a pair moving relative to the background nuclear medium (Alm et al. 1996). The maximal relative velocity for the case with no magnetic field decay is $\delta v_{\max }=\delta \Omega_{\max } r \sim 6.3 \times 10^{8} \mathrm{~cm} / \mathrm{s}$, where $r \sim 10^{6} \mathrm{~cm}$ and $\delta \Omega_{\max }$ is the maximal angular velocity departure (corresponding to the case where superfluid rotates at the initial angular velocity while the normal component is virtually at rest). In the case of magnetic field decay, the maximal departure is determined by the field decay constant and we find that $\delta v_{\max } \leq 10^{7} \mathrm{~cm} / \mathrm{s}$ in all cases. An order of magnitude estimate of the critical velocity at the nuclear saturation density $n=0.16 \mathrm{fm}^{-3}$ gives $2 \times 10^{8} \mathrm{~cm} / \mathrm{s}$ for $\Delta=1 \mathrm{MeV}$. When $v_{\max }>v_{\text {cr }}$ the condensate will lose momentum via the pair-braking processes until the angular velocity departure drops below the critical value. Some models might maintain the situation where $\delta v_{\max } \leq v_{\text {cr }}$ which would imply earlier and smaller "drops" of evolutionary tracks.

Observationally, our conjecture may be testable. First, if the currently measured braking index is significantly influenced by internal torques, then it should change over time in a way that can be tested statistically in a sample of pulsars (Cordes \& Chernoff 1997).

\footnotetext{
${ }^{2}$ For the neutron star model with canonical mass $1.4 M_{\odot}$ based on the AV14 equation of state of Wiringa, Fiks and Fabrocini (1988) we find $\alpha=0.094$, for the case when superfluidity persists through the core density region $\rho_{\min } \leq \rho \leq \rho_{c}$, where $\rho_{c}=1.03 \times 10^{15} \mathrm{~g} \mathrm{~cm}^{-3}$ is the central density.
} 
Second, the evolution towards the sector of millisecond pulsars in the $P-\dot{P}$ diagram does not imply increase of the mass of pulsar, while the pulsars spun-up in an accretion phase should be more massive than their non-spun-up counterparts by $\sim 0.1 M_{\odot}$. The duration of the spin-up phase in the present model for internal torques is the superfluid relaxation time, which e.g. for times $\sim 10^{6}$ yr implies that in a population of Gyr old millisecond pulsars there is a probability of $\leq 10^{-3}$ of observing a pulsar with $\dot{P}<0$.

To summarize, we have shown that weakly coupled superfluid in pulsars affects the secular dynamics of pulsars in two ways: (i) For constant magnetic field the internal torque causes an apparent degradation of the braking torque, thus mimicing field decay. The braking index, after the epoch of drastic enhancement, reduces to values $\sim 2.5$, close to those measured for young pulsars. (ii) In the case where the magnetic field is attenuated the star undergoes a spin-up phase after which it emerges as a solitary millisecond pulsar; one should note, however, that fits to the fastest known solitary millisecond pulsars require unusual parameter values which seem to be incompatible with the present view on the structure and field evolution of neutron stars.

We thank Ira Wasserman for helpful discussions. AS gratefully acknowledges the support of the Max Kade Foundation, New York (NY). JMC thanks the NSF for grant AST95-28394.

\section{REFERENCES}

Alm, Th., Röpke, G., Sedrakian, A., \& Weber, F. 1996, Nucl. Phys. A, 604, 491

Alpar, M. A., Chau, H.-F., Cheng, K. S., \& Pines, D. 1996, ApJ, 459, 706

Alpar, M. A., Langer, S. J., \& Sauls, J. A. 1984, ApJ, 282, 533

Baym, G., Pethick, C., Pines, D., \& Ruderman, M. 1969, Nature, 224, 872

Bhattacharya, D., \& van den Heuvel, E. J. P. 1991, Phys. Rep., 203, 1

Boyd, P. T., et al. 1995, ApJ, 448, 365

Chandler, E. \& Baym, G. 1986, J. Low Temp. Phys., 62, 119

Chen, K., \& Ruderman, M. 1993, ApJ, 402, 264

Cordes, J. M. \& Chernoff, D. F. 1997, ApJ, submitted (see also astro-ph/9707308) 
Kaspi, V. M., et al. 1994, ApJ, 422, L83

Khalatnikov, I. M. 1965, An Introduction to the Theory of Superfluidity (NY: W.A. Benjamin, Inc.), pp. 93-104.

Kulkarni, S. 1986, ApJ, 306, L85

Link, B., Epstein, R. I., \& Baym, G. 1993, ApJ, 403, 285

Lyne, A. G., Pritchard, R. S., \& Graham-Smith, F. 1988, MNRAS, 233, 667

Lyne, A. G., et al. 1996, Nature, 381, 497

Lyne, A. G., Manchester, R. N., and Taylor, J. H. 1985, MNRAS, 213, 613

Melatos, A. 1997, MNRAS, in press

Phinney, E. S., et al. 1988, Nature 333, 832

Romani, R. W. 1990a, Nature, 347, 741

-. 1990b, ApJ, 357, 493

Romani, R. W., Kulkarni, S., \& Blandford, R. D. 1987, Nature, 329, 210

Ruderman, M., Shaham, J., \& Tavani, M. 1991, ApJ, 336, 507

Ruderman, M. 1991, ApJ, 366, 261; ibid, 382, 587

Sauls, J. A., Stein, D. L., \& Serene, J. W. 1982, Phys. Rev. D25, 967

Sedrakian, A. D., et al. 1995, ApJ, 447, 324

Shibazaki, N., \& Mochizuki, Y. 1995, ApJ, 438, 288

Wiringa, R. B., Fiks, V., \& Fabrocini, A. 1988, Phys. Rev. C38, 101 